\title{
Safeguard Application Options for the Laser-Based Item Monitoring System (LBIMS)
}

\section{October 2008}

Prepared by:

M. D. Laughter

ORNL Global Nuclear Security Technology Division

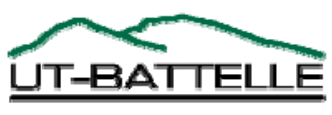




DOCUMENT AVAILABILITY
Reports produced after January 1, 1996, are generally available free via the U.S. Department of
Energy (DOE) Information Bridge.
Web site http://www.osti.gov/bridge
Reports produced before January 1, 1996, may be purchased by members of the public from the
following source.
National Technical Information Service
5285 Port Royal Road
Springfield, VA 22161
Telephone 703-605-6000 (1-800-553-6847)
TDD 703-487-4639
Fax 703-605-6900
E-mail info@ntis.gov
Web site http://www.ntis.gov/support/ordernowabout.htm
Reports are available to DOE employees, DOE contractors, Energy Technology Data Exchange
(ETDE) representatives, and International Nuclear Information System (INIS) representatives from
the following source.
Office of Scientific and Technical Information
P.O. Box 62
Oak Ridge, TN 37831
Telephone 865-576-8401
Fax 865-576-5728
E-mail reports@osti.gov
Web site http://www.0sti.gov/contact.html

\section{DISCLAIMER}

This report was prepared as an account of work sponsored by an agency of the United States Government. Neither the United States Government nor any agency thereof, nor any of their employees, makes any warranty, express or implied, or assumes any legal liability or responsibility for the accuracy, completeness, or usefulness of any information, apparatus, product, or process disclosed, or represents that its use would not infringe privately-owned rights. Reference herein to any specific commercial product, process, or service by trade name, trademark, manufacturer, or otherwise, does not necessarily constitute or imply its endorsement, recommendation, or favoring by the United States Government or any agency thereof. The views and opinions of authors expressed herein do not necessarily state or reflect those of the Unites States Government or any agency thereof. 
ORNL/TM-2008/086

Global Nuclear Security Technology Division

\title{
SAFEGUARD APPLICATION OPTIONS FOR THE LASER-BASED ITEM MONITORING SYSTEM (LBIMS)
}

\author{
Mark Laughter \\ Oak Ridge National Laboratory \\ P.O. Box 2008, MS-6315, Oak Ridge, TN 37831
}

Date Published: October 2008

Prepared by

OAK RIDGE NATIONAL LABORATORY

Oak Ridge, Tennessee 37831

managed by

UT-BATTELLE, LLC

for the

U.S. DEPARTMENT OF ENERGY

under contract DE-AC05-00OR22725 



\section{CONTENTS}

Page

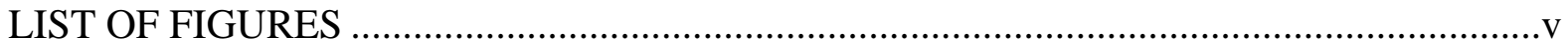

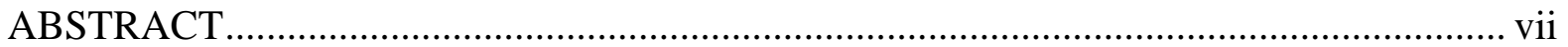

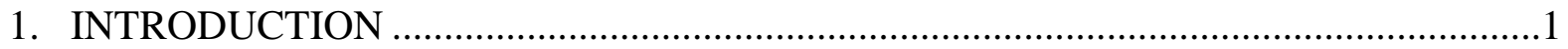

2. LASER-BASED ITEM MONITORING SYSTEM (LBIMS) ......................................

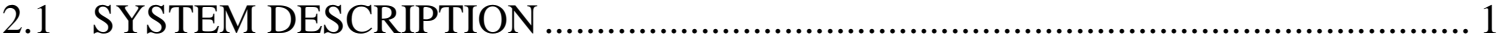

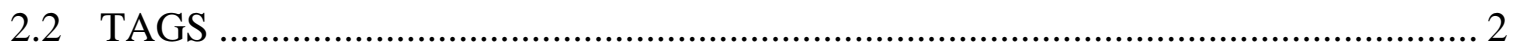

2.2.1 Generic Container Tags .......................................................................... 2

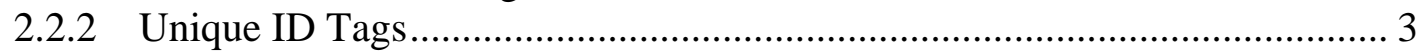

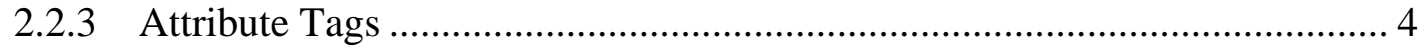

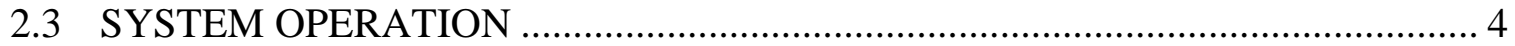

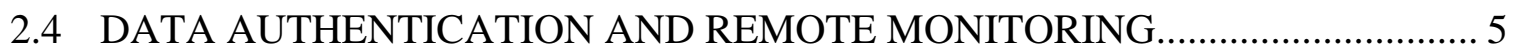

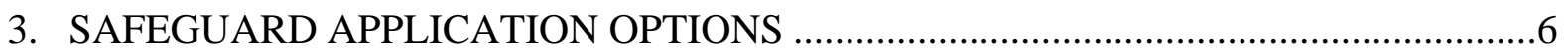

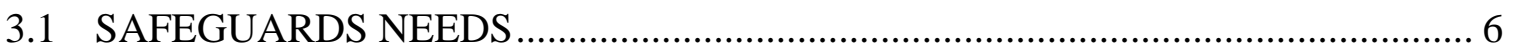

3.2 LBIMS APPLICATION OPTION 1-STATIC MONITORING .......................... 6

3.3 LBIMS APPLICATION OPTION 2-PROCESS MONITORING.......................... 7

3.4 LBIMS APPLICATION OPTION 3-MULTI-LAYERED INTEGRATION ......... 8

3.5 LBIMS APPLICATION OPTION 4-LINE-OF-SIGHT DATA TRANSFER........ 8

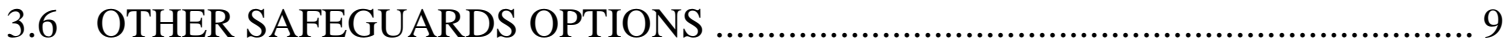

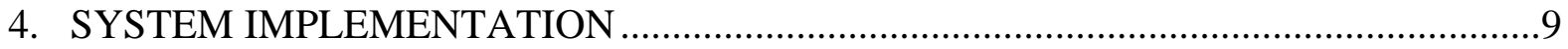

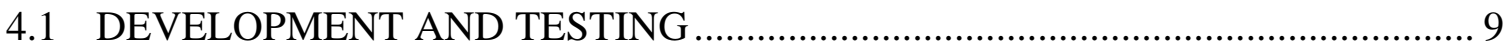

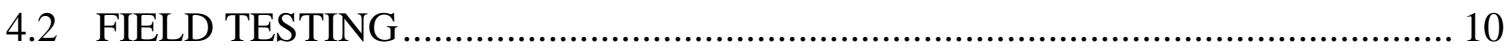

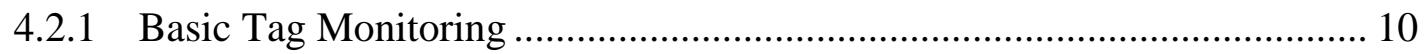

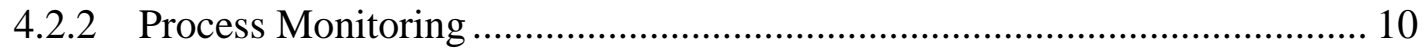

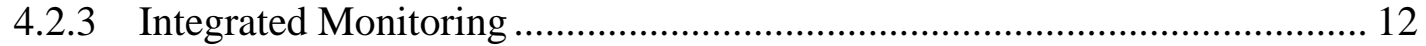

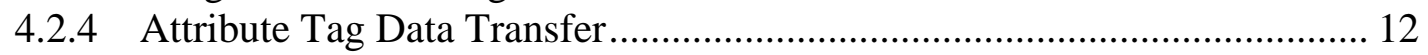

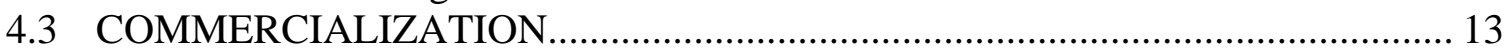

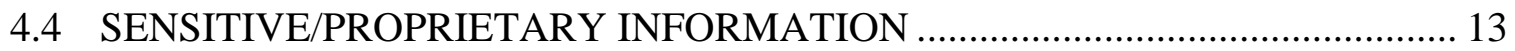

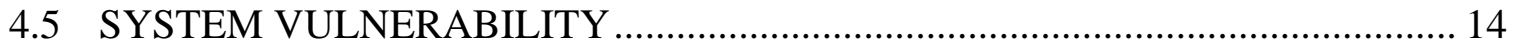

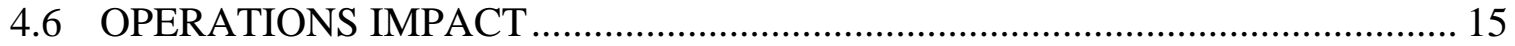

4.7 INTEGRATION WITH OTHER ADVANCED SAFEGUARD SYSTEMS.......... 15

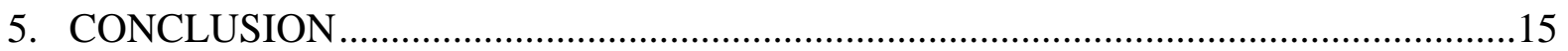





\section{LIST OF FIGURES}

Figure

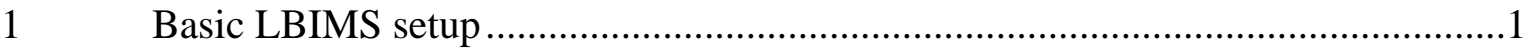

$2 \quad$ Prototype laser transceiver and power supply ..............................................2

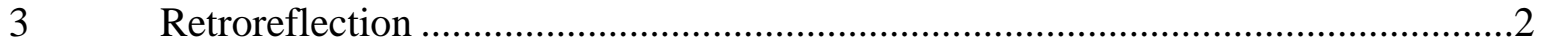

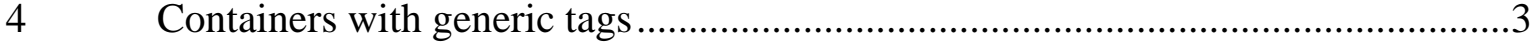

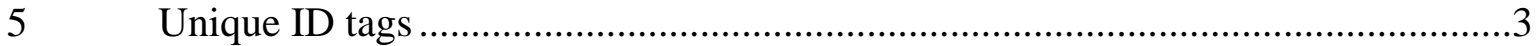

$6 \quad$ Prototype attribute tag/load-cell system........................................................4

$7 \quad$ Line-of-sight data transfer from sensor array ...................................................

$8 \quad$ Static-monitoring field trial..................................................................... 11

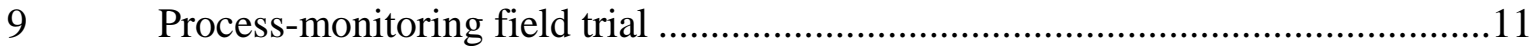

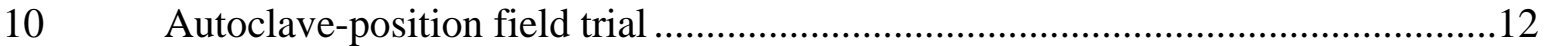





\begin{abstract}
Researchers at Oak Ridge National Laboratory (ORNL) are developing a Laser-Based Item Monitoring System (LBIMS) for advanced safeguards at nuclear facilities. LBIMS uses a low-power laser transceiver to monitor the presence and position of items with retroreflective tags. The primary advantages of LBIMS are its scalability to continuously monitor a wide range of items, its ability to operate unattended, its low cost of implementation, and its inherent information security due to its line-of-sight and non-broadcasting operation.

The primary proposed safeguard application of LBIMS is described in its name: item monitoring. LBIMS could be implemented in a storage area to continuously monitor containers of nuclear material and the area in which they are stored. The system could be configured to provide off-site notification if any of the containers are moved or removed or if the area is accessed. Individual tags would be used to monitor storage containers, and additional tags could be used to record information regarding secondary storage units and room access. The capability to register small changes in tag position opens up the possibility of several other uses. These include continuously monitoring piping arrangements for design information verification or recording equipment positions for other safeguards systems, such as tracking the opening and closing of autoclaves as part of a cylinder tracking system or opening and closing valves on a sample or product take-off line. Combined with attribute tags, which transmit information from any kind of sensor by modulating the laser signal, LBIMS provides the capability to wirelessly and securely collect safeguards data, even in areas where radio-frequency or other wireless communication methods are not practicable. Four application types are described in this report: static item monitoring, in-process item monitoring with trigger tags, multi-layered integration with trigger tags, and line-of-sight data transfer with attribute tags. Field trials for each of these applications are described.
\end{abstract}





\section{INTRODUCTION}

The International Atomic Energy Agency (IAEA) is charged with monitoring nuclear material in safeguarded facilities. With the expected global growth of the nuclear energy industry, this task will expand in the future and tax the IAEA's limited resources. To perform its job effectively and efficiently, the IAEA can benefit from the development of advanced safeguards systems - such as the Laser-Based Item Monitoring System (LBIMS) being developed at Oak Ridge National Laboratory (ORNL).

\section{LASER-BASED ITEM MONITORING SYSTEM (LBIMS)}

\subsection{SYSTEM DESCRIPTION}

LBIMS is a system developed by researchers at ORNL to monitor containers of nuclear material. The system uses a low-power laser transceiver to bounce signals off retroreflective tags. The basic system has four components: (1) a laser source, usually a low-power diode laser with a miniature scanning system that targets the beam; (2) a retroreflective tag, which has a microstructure that returns some scattered laser light back along its original path; (3) a photodetector to detect the retroreflected light; and (4) a computer system to operate the laser scanning system and register the results. This basic setup is shown in Fig. 1. A photograph of the prototype transceiver assembly and its power supply is given in Fig. 2.

Laser scanning is achieved by shifting the beam with two mirrors that pivot on perpendicular axes. The prototype system allows for optical angular deflection of $\pm 30^{\circ}$ on each axis, thereby allowing the system to scan multiple distinct items. The mechanical motion of the mirrors allows discrete adjustment of the laser position, which limits the system resolution along with the diameter of the laser beam. For the prototype system the angular resolution is $8 \mu \mathrm{rad}$, which is translated to an $x-y$ resolution dependent on the distance to the target. In other words, when the transceiver assembly is placed closer to the tag array, greater resolution is possible but less area can be scanned. At a $5 \mathrm{~m}$ distance, the system can cover

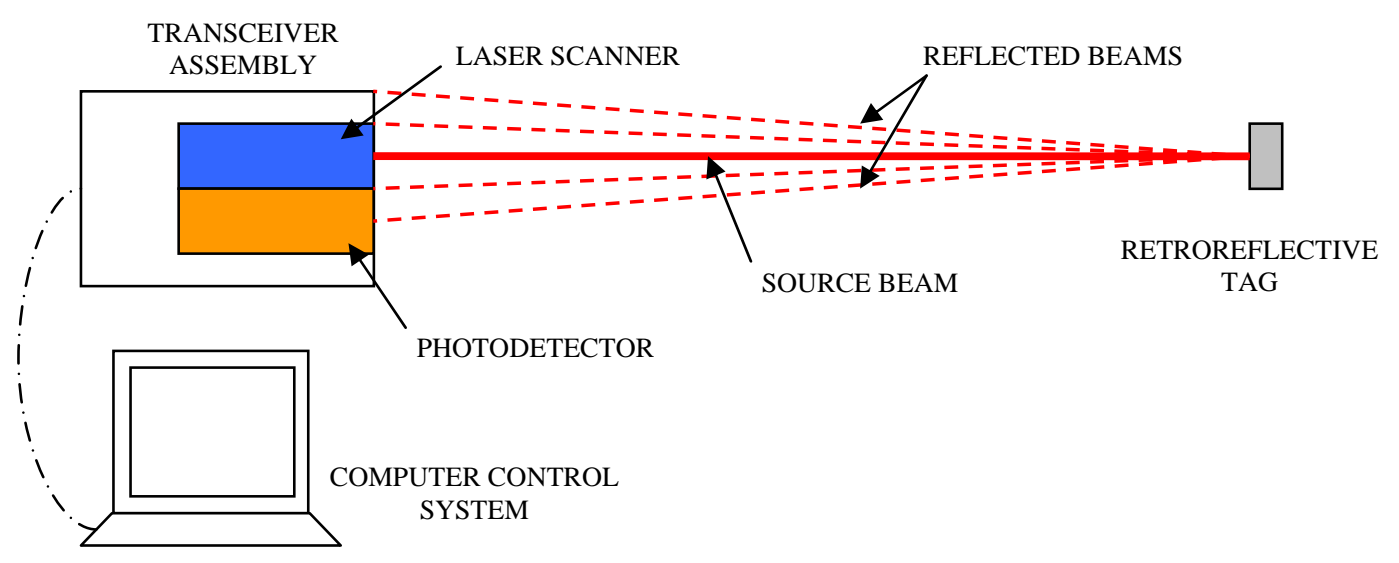

Fig. 1. Basic LBIMS setup. 


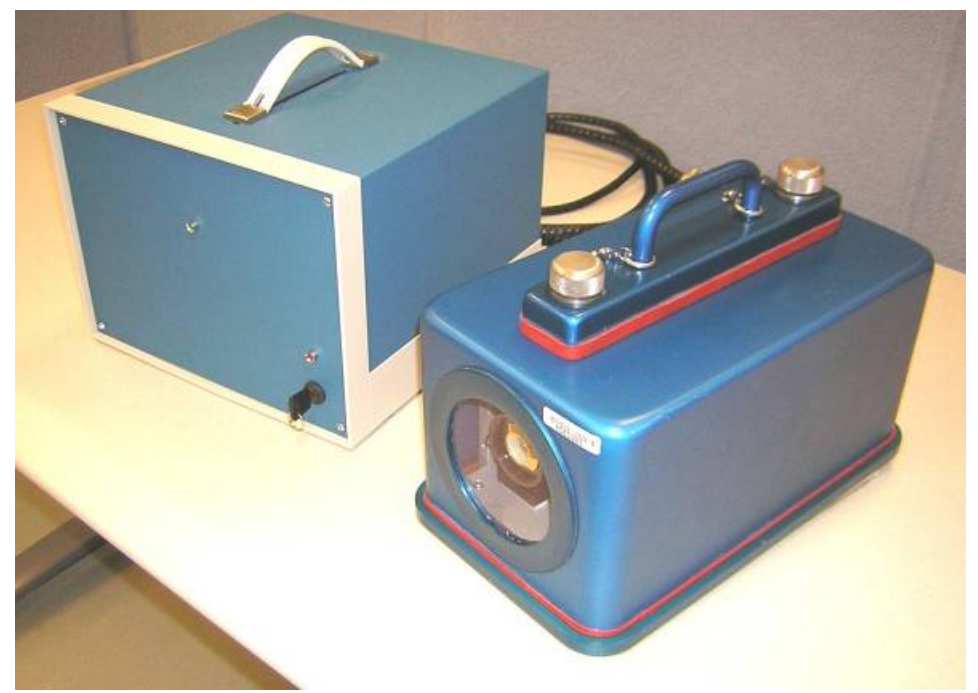

Fig. 2. Prototype laser transceiver and power supply.

just over $33 \mathrm{~m}^{2}$ with a resolution of $0.04 \mathrm{~mm}$, while at $20 \mathrm{~m}$, the system can cover over $530 \mathrm{~m}^{2}$, but with a resolution of $0.16 \mathrm{~mm}$. Depending on the setup, the system can scan approximately 1000 points per second.

\subsection{TAGS}

There are three types of tags currently used by the system: generic container tags, unique ID tags, and attribute tags. All of the tag types are made of commercially available retroreflective material. This material has a microstructure that reflects some scattered laser light back along its original path regardless of the angle of incidence, as opposed to the traditional angle of reflection afforded by a simple mirror. This concept is illustrated in Fig. 3. Use of this material for the tags allows the laser to scan a wide area without the need for the tags to be perpendicular to the beam.

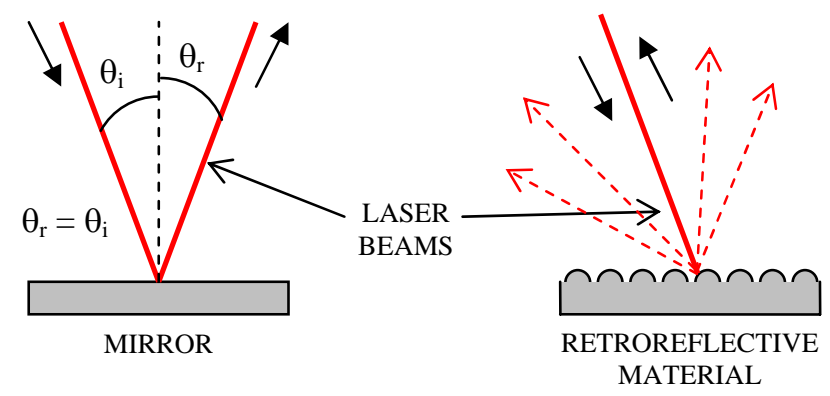

Fig. 3. Retroreflection.

\subsubsection{Generic Container Tags}

The generic container tags are simply pieces of retroreflective material with an adhesive backing that can be affixed to any reasonably smooth container or surface. The system has no means to directly distinguish between generic tags. However, position sensitivity allows 
the system to distinguish if one tag has replaced another, by reporting that the original tag has changed position. It should be stressed that this does not indicate anything directly about the container to which the tag is attached; the position sensitivity only allows the system to infer the movement of the container based on the movement of the tag. Figure 4 shows an array of containers bearing generic tags. (The tags appear as light patches on container surfaces, since the tags also reflect the ambient light in the room towards the camera.)

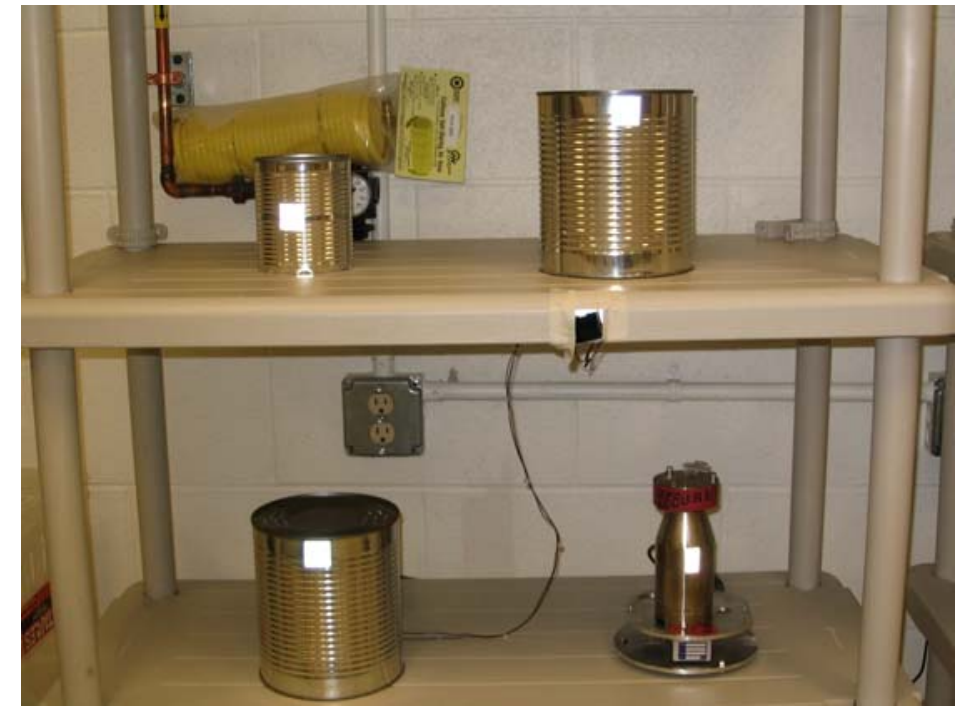

Fig. 4. Containers with generic tags.

\subsubsection{Unique ID Tags}

Unique ID tags are generic tags that have a unique pattern blacked out, so that areas of the tag are nonreflective. The specific pattern of reflective and nonreflective areas allows the system to log that tag with a unique ID and recognize it in the future, even if it changes position or orientation. These tags provide a "barcode-at-a-distance" capability (as termed by the system developers).

Currently, unique ID tags are constructed by applying a nonreflective decal to a square of retroreflective material, as shown in Fig. 5. These preliminary tags have a relatively large

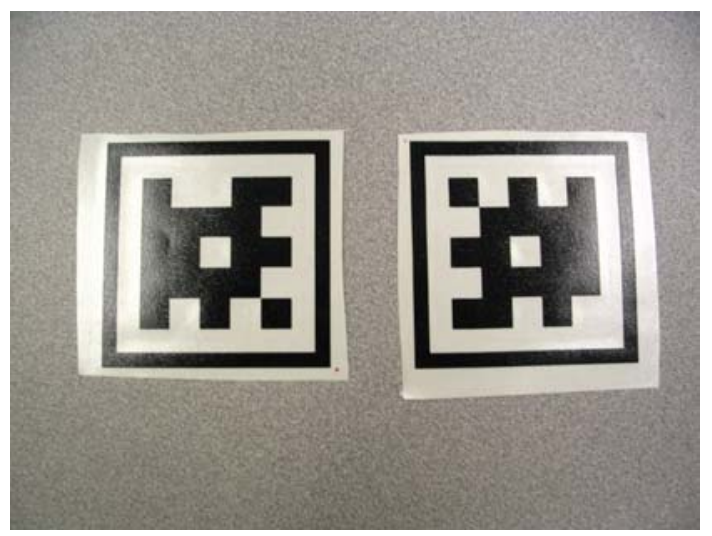

Fig. 5. Unique ID tags. 
scale pattern that can be counterfeited easily, so a more advanced unique ID tag design must be developed to reduce vulnerability to forgery. This could involve either a smallerresolution pattern or even a random "scratch" pattern; in either case, if the pattern is not sufficiently irreproducible, it loses all value as a unique identifier.

\subsubsection{Attribute Tags}

Attribute tags make use of a liquid crystal shutter to modulate the reflectivity of a generic tag. The modulation control system is connected to a sensor that monitors the attribute of interest. The pattern of modulation is monitored by the transceiver system and can convey any type of information. The simplest attribute tags transmit a simple value, such as a temperature reading or load-cell measurement. Figure 6 shows a prototype attribute tag and the modulation system (disassembled) to connect it to a load cell. With further development, attribute tags could be designed to transmit more complex information; the information could be encrypted so that even someone with line-of-sight access to the tag could not readily access the data.

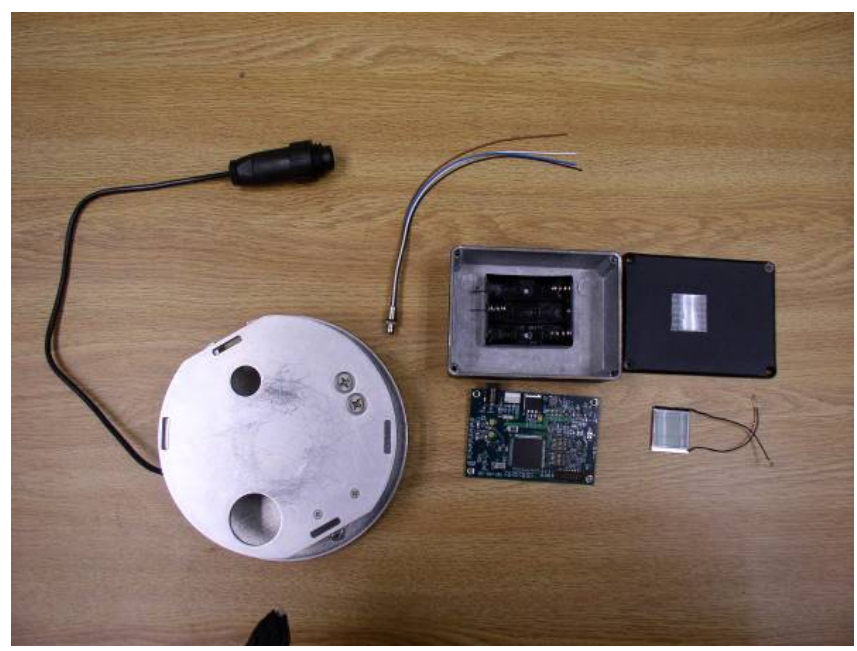

Fig. 6. Prototype attribute tag/load-cell system.

\subsection{SYSTEM OPERATION}

LBIMS has two primary phases of operation: (1) establishment of a "baseline" tag configuration for the scan area and then (2) continuous monitoring of the scan area to detect changes from that baseline configuration. The baseline phase begins with the system operator using the transceiver to act as a laser pointer to define the scan area boundaries. (Alternately, the operator could position tags at the edges of the scan area and define those tags in the system as "boundary tags", thus allowing the transceiver to be moved or jostled slightly without the need to acquire a new baseline scan.) The operator then instructs the system to "build array", which causes the system to automatically scan the bounded area, locate all retroreflective tags, and then scan each found tag to determine its precise position. If the system locates any tag that appears to be a unique ID tag, it scans that tag more precisely to determine its ID pattern and to log that tag pattern in its memory. The system 
also allows the operator to manually identify unique ID and attribute tags from those found in the initial scan.

In the continuous-monitoring phase, the system constantly and randomly cycles through all the tags it has identified to confirm continued presence. Periodically, the system randomly selects individual scans to undergo detailed scan to detect any change in position. In effect, this confirms not only the precise position of the tags but also confirms the presence or absence of non-retroreflective surfaces. Without these random detailed scans, the system could be defeated by shielding the entire scan area with a retroreflective screen. In addition, the system dwells on any attribute tags to collect the most recent data it is presenting. The system may also be programmed to rescan the entire bounded area periodically to find any additional tags that may have appeared.

For the monitoring phase of operation, data generated by the system are a simple array of “yes/no" parameters. For each tag, it confirms its continued presence and continued position compared with its baseline configuration and then registers an alert if there is a deviation in either of these parameters. The software is programmed with thresholds that define when a series of alerts (individual deviations) warrant an alarm (event log, notification, response, etc.). For any unique ID tag, the system confirms that the ID tag is the same as the original tag, and for any attribute tag, it periodically registers the data presented from that tag.

More-complex operation could be made possible by programming the system to use individual tags as triggers for further action. For instance, if a tag is placed on the closed door of a cabinet, opening the cabinet could trigger the system to immediately scan the nowexposed area of that cabinet to look for additional tags. Similarly, a tag placed on a door to a storage room could trigger the system to rescan the room every time the door is opened. Complex systems can be constructed by using tags as “yes/no" triggers in this way. Even more complex systems could be created by using attribute tags as triggers. Instead of just "yes/no" commands, an attribute tag could convey a broader range of instructions to the system.

\subsection{DATA AUTHENTICATION AND REMOTE MONITORING}

The computer control system can be operated remotely, although the system's initial setup and tag placements obviously must be completed at the site. Even setting the scan area could be done remotely, within a limited range, if a webcam were used to monitor the laser pointer during boundary adjustment. Larger readjustments (e.g., extending the boundary beyond the scan-able range or moving the transceiver assembly) would have to be completed at the site. If monitoring multiple areas is required, multiple transceiver assemblies could be used and controlled by a single computer system. In the continuous-monitoring phase, alerts, alarms, and other data could be time-stamped, stored locally, and/or transmitted off-site. The typical data volume is very small since the data in the simplest configuration are just an array of “yes/no” parameters.

Standard data encryption and authentication techniques could be used to protect data communicated between either the computer control system or an on-site data aggregator and the off-site receiver. The data collected by the system is inherently self-authenticating since 
the system compares all subsequent measurements with its scanned baseline configurationexcept in the case of data collected from attribute tags. Additional authentication could be obtained by placing a unique ID tag in the system's scan area (but not on a container) and then instructing the system to confirm system operation by occasionally scanning that tag. However, this would be redundant with an inherent feature of the system's designed function. On the other hand, in the cases of process monitoring and attribute tags, such authentication may be useful if the environmental conditions of the scanned area could change over time (for instance, moisture or temperature that degrade the reflected signal), and a known tag is used to calibrate the signals from other tags.

For attribute tags, data authentication would take place both at the sensor/tag interface and in the encryption for tag-to-transceiver signals. However, the limits of the data collected by LBIMS necessitate caution in interpreting the results. For instance, an LBIMS monitoring an attribute tag connected to a load cell would not be able to distinguish between the mass change of a container being filled through legitimate operation and someone illicitly adding weights to the load cell. This limitation, common to many automated safeguards systems, is overcome by layering different systems to cover the "blind spots" and provide confirmatory information using other monitoring technologies.

\section{SAFEGUARD APPLICATION OPTIONS}

\subsection{SAFEGUARDS NEEDS}

The IAEA is expected to perform its safeguarding and verification functions on an increasing number of nuclear facilities and growing amounts of nuclear material without commensurate increases in funding and personnel resources. Therefore, the IAEA has emphasized the need for developing technologies that are both effective and efficient in carrying out these functions. Systems that can operate in an automated fashion and deliver their results remotely are especially advantageous, because they both reduce the travel required for inspectors to visit facilities and simplify the analysis of data by inspectors at the Agency's headquarters. Continuous-monitoring systems are also beneficial in providing continuity of knowledge between inspections and reducing the time required to perform periodic physical inventories.

\subsection{LBIMS APPLICATION OPTION 1-STATIC MONITORING}

The primary proposed safeguard application of LBIMS is described in its name: static item monitoring. LBIMS could be implemented in a storage area to continuously monitor containers of nuclear material or other items of interest. The system could be configured to provide off-site notification if any of the containers are altered or moved. Generic tags would be used to monitor storage containers, and additional tags could be used to record information on secondary storage units and room access, such as opening cabinets and doors.

This application would be most useful at dedicated storage facilities, where nuclear material or controlled components are expected to be stored undisturbed for a long period of time. In 
such a facility, where a manual inventory is the only method to verify continued presence of the controlled items, on-site inspections must be frequent enough to detect diversion within a useful time frame. A continuous-monitoring system such as LBIMS would allow such inspections to be less frequent or eliminated altogether except when the system alarms.

As described above, the basic static monitoring system can be made more complex or robust by the addition of trigger tags, for instance where the system is triggered to rescan the original area or scan different areas based on the position of tags on cabinets and doors. In addition, attribute tags can be used to provide another layer of static monitoring of a container's contents. For a container placed on a load cell, an attribute tag will report not only the container's presence but also the continuity of the measured mass. Similarly, a radiation detector near a group of containers can report continuity of the radiation field produced by the contents of those containers.

\subsection{LBIMS APPLICATION OPTION 2-PROCESS MONITORING}

LBIMS also can be used as a continuous process monitoring tool in areas where items or containers, such as $U_{6}$ cylinders or sample containers, move along a regular path. The system could operate as a laser-based motion detector by triggering when its beam path has been interrupted. This, in turn, could trigger the system to scan a set area and look for any new tags, thereby registering the presence of an item that has been temporarily placed in its scanning area. In this mode, the system would not be alarming every time a tag is found and then moved; it would only be logging and time-stamping the presence of tags.

Unique ID tags are especially useful for this option, because the items will be present only temporarily and not remain in precisely the same position every time. An example of the use of LBIMS in this way would be a temporary $\mathrm{UF}_{6}$ cylinder holding area. LBIMS would monitor the entrance and exit paths to the holding area and scan the entire area for the unique ID tags of each cylinder whenever it is triggered. This would provide a continuous database of exactly which cylinders are present in the holding area at a given time and record when each cylinder was brought into or removed from the holding area. If interconnected LBIMS transceivers are used to monitor a series of in-process storage areas, rules-based logic could be used to determine that all cylinders are accounted for and travel through the plant in the appropriate sequence and on a reasonable schedule.

The LBIMS static monitoring capability also could be useful in process monitoring, for instance, in monitoring piping arrangements for design information verification. Instead of having to visually inspect piping arrangements and compare them with operator-controlled photographs, an inspector could have the operator affix small generic tags to several locations on the pipes and then instruct an installed LBIMS to scan the area and continuously monitor to ensure that the arrangement has not changed. The operator would retain the capability to reconfigure the piping for legitimate purposes but must notify the inspector who will use the LBIMS to set a baseline for the new configuration. This application would be useful in monitoring any static arrangement, such as secondary feed and withdrawal points (e.g., valves on product sampling lines) for an enrichment cascade. 


\subsection{LBIMS APPLICATION OPTION 3-MULTI-LAYERED INTEGRATION}

Another possible use of LBIMS in process monitoring is as a single layer in a multicomponent safeguards system. LBIMS can be used to trigger actions in other systems, such as detecting the presence of a cylinder to trigger a radiation detector to take a reading or indicating when the doors are opened or closed on an autoclave to provide continuity of knowledge for a cylinder tracking system. LBIMS provides a low-cost capability to reduce the data volume for other continuous systems and thereby removes the need for facility operator action for the systems to function.

\subsection{LBIMS APPLICATION OPTION 4-LINE-OF-SIGHT DATA TRANSFER}

Another possible use of LBIMS is as a secure wireless data transfer system. In an area with distributed data-collection systems, such as an array of radiation detectors, there may be no expedient way to run the wiring needed to collect the information from the individual sensors, and operator restrictions or security concerns may preclude the use of radiofrequency or other wireless communication methods. If, as shown in Fig. 7, each sensor is coupled with an attribute tag that is positioned in the line of sight of an LBIMS transceiver, the data could be aggregated securely for collection in a mailbox system or for transfer offsite to the inspector. However, the line-of-sight requirement may be difficult to maintain in an operating facility. This application may be most useful as a secondary data-transfer method to provide independent authentication and protect against interruption of a primary data feed.

The current system can scan approximately 1000 points per second. This allows a maximum information transfer rate of about one kilobit per second (kbps), which is comparable to early voice-band modems from the 1960s and 1970s. This transfer rate would be enough to continuously monitor several attribute tags with constantly changing values, such as load-cell readings at feed and withdrawal stations.

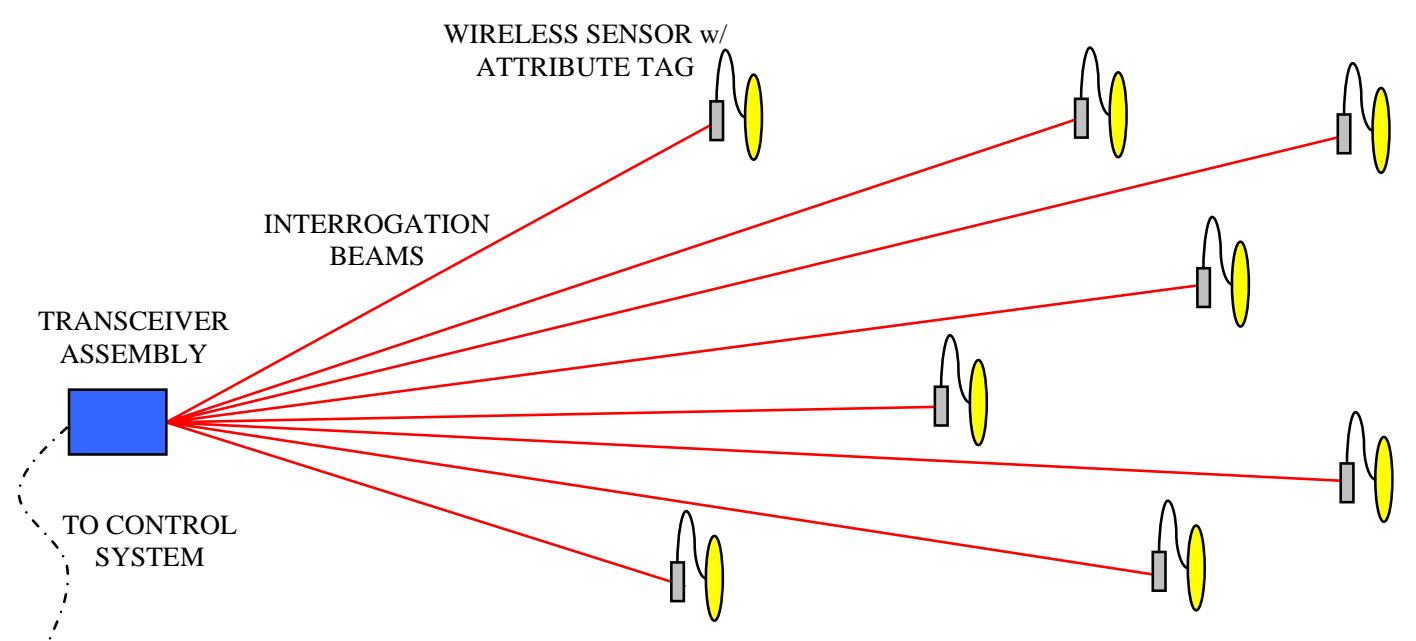

Fig. 7. Line-of-sight data transfer from sensor array. 


\subsection{OTHER SAFEGUARDS OPTIONS}

There are numerous other possible uses of LBIMS as a safeguards system, although most will fall into one of the four categories described above. In a larger sense, LBIMS could be a valuable tool in a facility-wide process monitoring system-incorporating load cells, flow monitors, and other advanced technologies. LBIMS could be used in all the applications discussed above in a single facility: static monitoring, process monitoring, triggers for other systems, and line-of-sight data transfer. In this respect, LBIMS would be more than a distinct safeguards system and become a common tool available to any system.

\section{SYSTEM IMPLEMENTATION}

The possible uses of LBIMS are numerous, but the actual benefit of the system will depend on its effectiveness and efficiency when implemented at an operating facility. For efficiency, LBIMS must either affordably perform a function that cannot be accomplished by another system or replace another available system at a lower cost. In addition, LBIMS must be scalable to different facility types and sizes to limit the impact of development costs on a perimplementation basis. For effectiveness, LBIMS must operate reliably and safely with minimal impact on facility operations or false alarms and must provide assurance to the facility operator that sensitive or proprietary information will not be compromised.

\subsection{DEVELOPMENT AND TESTING}

While LBIMS in its simplest form is being commercialized, more-advanced uses of the system, which provide more capabilities and customizability, are in a laboratory development phase. The current setup uses LabVIEW software to control the system and log the results.

Current development efforts focus on determining operating limits of the system. This includes environmental testing of the transceiver to determine its response to temperature changes and moisture. Initial experiments show that the laser output varies with ambient temperature, causing false displacement errors until the system is thermally stabilized. Additional testing of system limits will include determining the system resolution (e.g., how fine a pattern can be used for the unique ID tags) and sensitivity to position of the retroreflective tags (e.g., how far a tag can be rotated before it cannot be seen by the system).

The software to program and operate an LBIMS requires significant development before it is ready for operational deployment. For implementation, control software needs to be developed that is exportable with the system and is more compact and efficient than a large software suite such as LabVIEW (which includes many functions that are not needed for this system). This software will include a means to communicate with multiple transceivers, a user interface that allows customizing for specific applications (e.g., programmable triggers), and appropriate remote monitoring and authentication capabilities. This software must be simple to use, but robust-if LBIMS is to see wide-scale implementation. Not only does the user interface and customizability need to be improved, but additional layers for signal authentication and data encryption also need to be added. Furthermore, to function in an 
operating facility, the system must be fine-tuned to minimize nuisance alarms while providing sufficient confidence in the continuity of knowledge of the monitored items. For some applications, the settings needed to reduce the nuisance alarms to an acceptable level may make the system ineffective; a useful signal may become lost in the noise.

\subsection{FIELD TESTING}

Laboratory testing of LBIMS is ongoing, including long-term continuous monitoring of containers in the lab. However, field testing in an operating facility is necessary to demonstrate to facility operators and the IAEA that the system is effective. A separate field test for each application option described above would be appropriate.

\subsubsection{Basic Tag Monitoring}

One field test that should be given higher priority because it demonstrates the basic LBIMS function would involve monitoring items in a low-traffic storage area over a period of weeks or months. The ideal facility would have a storage room that contains protected items arrayed on a shelf or otherwise visible. This room would normally be closed and remain undisturbed for long periods of time. Sufficient space would be required to set the LBIMS transceiver at a distance that allows the entire testing inventory to be scanned, and there must also be space for a stand or cabinet to hold the computer control system. An ethernet connection or some other communications port would allow remote monitoring but is not necessary for initial field trials. Tags would be placed on the items or containers. If the adhesive backing presently used is not acceptable to the facility operator for any reason, some alternative methods of attaching the tags would be devised. The system would perform its baseline scan and then begin monitoring continuously for a period of weeks or months, with someone occasionally checking just to make sure the system is still powered and operating. Generic tags would make up the bulk of this trial, but a handful of unique ID tags could be used as well, and an attribute tag connected to a simple sensor such as a temperature sensor would allow data to be collected on that application as well. Figure 8 is a basic diagram of the layout for this field trial.

\subsubsection{Process Monitoring}

To test the continuous process monitoring, a field trial should be performed in a manner similar to the above trial. However, the test should be performed in a temporary storage area where containers of some sort are brought in, held for a time, and then taken out. For this test, unique ID tags exclusively should be used on containers. As above, some alternative tag attachment methods could be devised if required by the operator. In addition, some trigger tags would be required to indicate when an item is taken into or out of the storage area and thus prompt the system to rescan the entire area to find new tags; generic tags would be used for these triggers. Depending on how items are stored in this area, the transceiver may have to be mounted in an elevated position or even directly overhead. The researcher will either attach unique ID tags to each item that may travel through the storage area during the test or provide a number of tags to the facility operator to place on items before they are taken into the storage area. Figure 9 shows a diagram of a possible layout for this trial. 


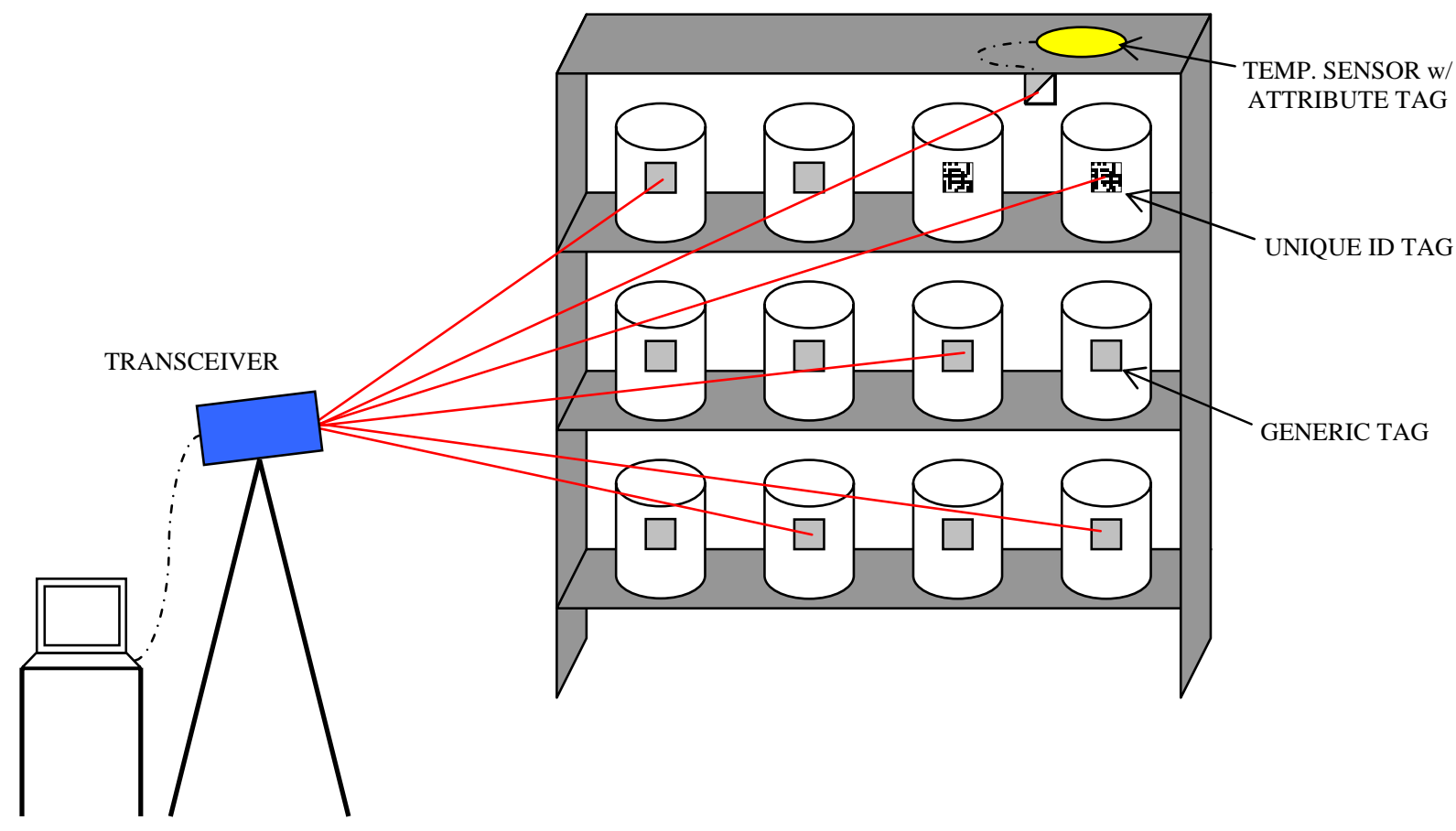

Fig. 8. Static monitoring field trial.

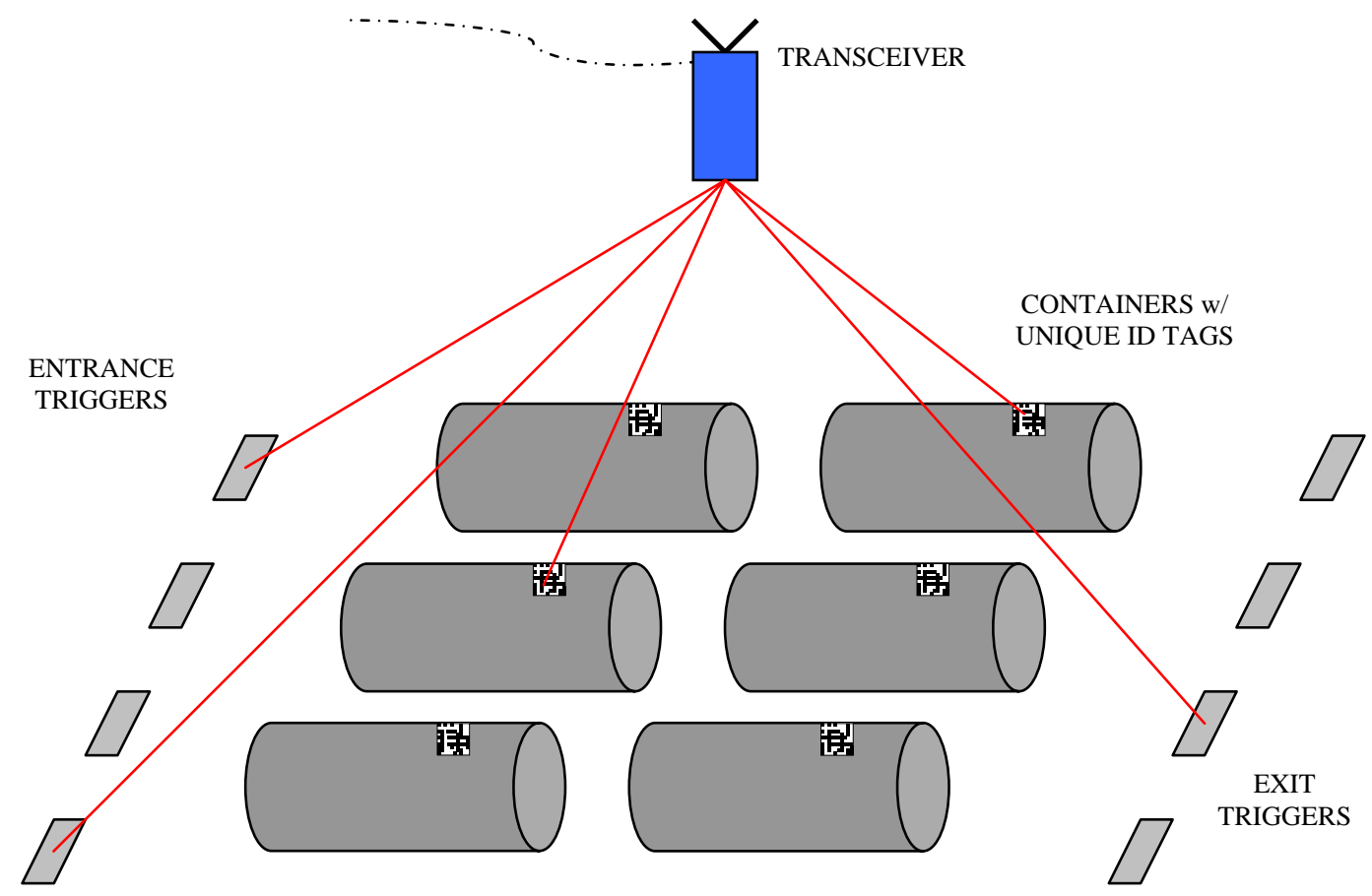

Fig. 9. Process monitoring field trial. 


\subsubsection{Integrated Monitoring}

A field trial of the third proposed application, multi-layered integration, would be to monitor a single variable in a process area involved in other safeguard system field trials. For instance, as an adjunct to the radio-frequency cylinder tracking system being tested at the Portsmouth Gaseous Diffusion Plant, an LBIMS could be set up near the autoclave to monitor tags on the autoclave lid, tracking whether the autoclave is open or closed. A number of small transceivers could be mounted relatively close to the autoclaves, so that only a small area on each is scan-able. Two unique ID tags would be placed on the autoclave lid: one that would fall within the scan area when the lid is closed, and one that would be scanned when the lid is open. The system would continuously monitor whichever tag was in its scan area, to confirm the continued open or closed state. When the system "loses" the tag (i.e., when the door is moving in either direction), the system would wait an appropriate amount of time for the motion to be completed and then rescan for the tag that confirms the lid is in the new position. This trial could be conducted concurrently with other field trials proposed for this area at the Portsmouth facility; computer equipment and ethernet cables are already in the appropriate locations. Figure 10 shows a diagram of one possible layout for this test. Alternatively, a single transceiver could be positioned to monitor a number of autoclaves; this would require defining different boundary regions within the scan area so that the system could determine how tag locations relate to autoclave operations.

\subsubsection{Attribute Tag Data Transfer}

The fourth application option, wireless data transfer with attribute tags, can be field tested in conjunction with the first trial described above. A simple sensor such as a digital thermometer can be attached to an attribute tag and then monitored continuously along with the items being observed. A more elaborate field trial could be held in conjunction with field
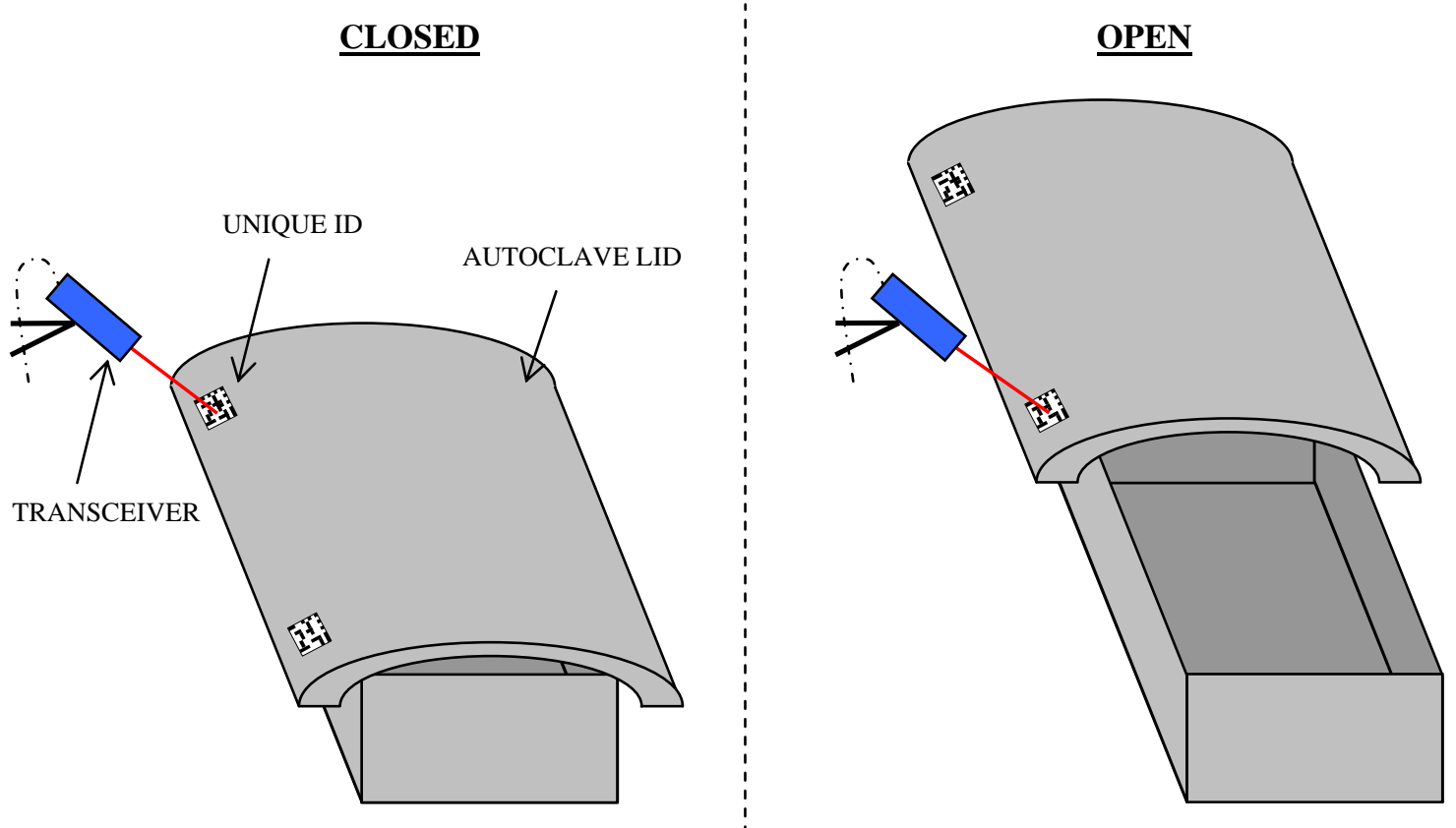

Fig. 10. Autoclave-position field trial. 
trials of other proposed safeguards systems, such as by using attribute tags to transmit data from a number of load cells during a field trial for the continuous load-cell monitoring project. In this situation, LBIMS could be used as a secondary data-transfer method (each load cell would be wired in the normal way, in addition to utilizing an attribute tag). Thus, the integrity of the other field trial will not be compromised if something goes wrong with the LBIMS trial.

\subsection{COMMERCIALIZATION}

LBIMS is in the process of being commercialized by Canberra. To be most useful for applications of different proportions, commercialized LBIMS transceivers should come in a range of sizes, based on the desired resolution and scan area. Low-resolution, small-scanarea transceivers would be used in applications such as triggers for multi-layered integration as described above, where the system is only looking for a tag in a small area at a relatively close distance. Container monitoring systems could be larger, to provide better coverage of a storage area and allow use of finer-resolution unique ID tags. Transceivers for line-of-sight data transfer should be sized to cover all of the attribute tags under their jurisdiction and may require higher-resolution and more-powerful lasers to provide good results over longer distances. If LBIMS is commercialized as a single “one-size-fits-all” system, its utility for multi-layered integration may be reduced and its cost may be prohibitive for many of the proposed applications.

Control systems for these commercialized transceivers should be small and self-contained, with the capability to communicate with an on-site data aggregator. Ideally, a system would be programmed for its specific task and then disconnected from the programming computer and allowed to perform its function-only sending its results to an aggregator and not relying on a connected personal computer for control.

If these capabilities are commercialized, a complete LBIMS, including transceiver, control system, software, and tags, should cost only on the order of thousands to tens of thousands of dollars, depending on the specific application.

\subsection{SENSITIVE/PROPRIETARY INFORMATION}

Operators are often concerned with limiting access to sensitive or proprietary information in their facilities. Any implementation of a safeguards system, such as LBIMS, must be negotiated to ensure that such information is protected. The line-of-sight portion of the LBIMS (between the transceiver and tags), which uses only visible light and has no capability to broadcast, is inherently secure since it would be extremely difficult to intercept the signal without alerting the system. (A complex scheme involving beam-splitters or fiber optics is conceivable, but very unlikely.) Furthermore, LBIMS provides innate information security since it monitors only the physical presence and movement of its tags and has no capacity to collect other types of information such as imagery. For static item monitoring situations using generic or unique ID tags, LBIMS neither requires nor provides any information on the contents of the containers it monitors. For generic tags in configuration monitoring, such as monitoring piping arrangements for design information verification, 
LBIMS only compares the position of its tags with their original positions. Thus, it does not store or transmit any sensitive information.

One solution to protect sensitive information in other safeguards systems is to program the system to transmit only "go/no-go" signals off-site, signifying whether the monitored attribute is inside or outside a predetermined range. This feature is inherent to the design of LBIMS using generic or unique ID tags, where it collects only "yes/no" information on tag position compared with the baseline scan.

When used for data transfer with attribute tags, the information sensitivity is dependent on what sensors the attribute tags are connected to, not the LBIMS itself. Yet even in these cases, the line-of-sight restriction provides an added layer of information security, because it would be difficult (if not impossible) for someone to intercept or spoof without detection the information being transmitted. In general, the protection of sensitive information is much easier with LBIMS than with comparable item monitoring systems such as video surveillance.

\subsection{SYSTEM VULNERABILITY}

Before LBIMS is considered for implementation, it must be subjected to a comprehensive and independent vulnerability assessment. If any aspect of the system can be easily manipulated, spoofed, or counterfeited by the operator, then it is not an effective safeguards tool. Every aspect of the system must be considered:

- Is it possible to access the contents of tagged containers without alerting the system?

- How difficult is it to forge duplicate tags?

- Can tags be removed and reattached without alerting the system?

- Because the system compares current scans with a baseline scan, is it possible to intercept the signal and loop the baseline signal in order to blind the system?

- Is there room in the margins of system sensitivity to perform illicit activities without triggering an alert?

These are just some of the questions that must be considered in the vulnerability assessment, which must include evaluation of the system as a whole, the individual components, and the data protocol. Vulnerabilities should be addressed effectively as early in the design phase as possible to limit the risk of increased implementation costs at the back end.

Even if the system works effectively and securely, placing too much reliance on one system increases the risk of having a single point of failure. If a single transceiver assembly is being used to monitor both container motion (via a generic tag) as well as container mass (via an attribute tag), then loss of the transceiver indicates complete loss of continuity of knowledge for that container. Any safeguards system must have redundancy to be effective; however, if too many layers of redundancy are required, then the approach may not be efficient in terms of costs and resources. 


\subsection{OPERATIONS IMPACT}

The design and implementation of LBIMS must ensure that the impact on facility operations is minimal. Tags should be attached to containers or positioned in facilities in such ways that ensure no impact on operations. The only significant safety consideration for facility operators should be the use of lasers. The laser used in the prototype LBIMS transceiver is a low-powered red diode laser that is classified as $3 \mathrm{R}$ (output power $<5 \mathrm{~mW}$ ) according to ANSI standards, the same classification as many laser pointers. Class 3R lasers are generally considered safe, but there is some risk of injury from prolonged exposure directly into the eye. The natural human blink reflex is normally sufficient to prevent injury from lasers that have this power output. For many of the suggested LBIMS uses, the system can be configured to provide additional engineering controls to preclude injury from the laser. For example, the transceiver can be positioned so that the beam remains overhead or well below the normal eye-level range. Since the system can scan approximately 1000 points per second, the scan frequency can be set so that the beam does not dwell too long in one specific direction, eliminating the risk of prolonged exposure. For static storage areas where the scan area may cross eye level, trigger tags (e.g., on the door to the storage room) could cause the system to shorten the dwell time when someone enters the room. Finally, in higher-traffic situations where the beam must be at eye-level, appropriate warning signage and perhaps even eye protection equipment are suggested as adequate administrative controls. For safety reasons, lasers of higher classification (3B or above) should not be incorporated into LBIMS.

\subsection{INTEGRATION WITH OTHER ADVANCED SAFEGUARD SYSTEMS}

As discussed above, LBIMS could be used as a component in other safeguards systems to provide triggers and continuity of knowledge. In a larger sense, LBIMS could be a valuable tool in a facility-wide process monitoring system, incorporating load cells, flow monitors, and other advanced technologies. LBIMS could be used in all the applications discussed above in a single facility: static monitoring, process monitoring, triggers for other systems, and line-of-sight data transfer. In this respect, LBIMS would evolve from being a distinct safeguards system and instead become a common tool available to any system-just as ethernet routers or video cameras are not considered independent systems but components in a larger scheme.

\section{CONCLUSION}

With the expected growth of the nuclear energy industry, the task of monitoring safeguarded nuclear material will expand in the future and tax the IAEA's limited resources. To perform its job effectively and efficiently, the IAEA can benefit from the development of advanced safeguards systems such as LBIMS. The IAEA is currently investigating laser technology for other safeguards applications, including the 3-D Laser Range Finder (3DLR) for design information verification and the Laser Item Identification System (L2IS) for $\mathrm{UF}_{6}$ cylinder "fingerprinting". LBIMS, being developed by researchers at ORNL, fits in with this trend towards laser-based safeguard technologies. 
LBIMS uses a low-power laser transceiver to monitor the presence and position of items with retroreflective tags. Three categories of tags are used: generic container tags, unique ID tags, and attribute tags. The primary advantages of LBIMS are its scalability to continuously monitor a wide range of items, its ability to operate unattended, its low cost of implementation, and its inherent information security due to line-of-sight restrictions.

This report describes four proposed types of safeguards applications for LBIMS: static item monitoring, in-process item monitoring with trigger tags, multi-layered integration with trigger tags, and line-of-sight data transfer with attribute tags. Broad field trials for each of these applications are described, although the system is still in a laboratory testing phase.

Canberra has licensed the system for commercial development. Provided this commercialization is efficient, the cost of implementation at nuclear facilities will be relatively low, and the system will be scalable to the size of the task. The operator impact of implementing LBIMS is largely restricted to safety issues concerning lasers, although the laser currently used by the system is not sufficiently powered to cause significant risk of injury.

In the long term, LBIMS could be developed into an effective tool for limited use with many safeguards applications - in addition to serving as an independent system for continuous static item monitoring. However, as it exists now, the system is not directly applicable as a safeguards system. LBIMS basically gives detection of tag movement that, in itself, does not translate to item safeguarding unless the tag is integrated with additional measures that provide assurance of containment, such as tamper-indicating seals. In addition, some of the applications proposed in this report, such as process monitoring and data transfer, may have simpler solutions in many cases.

The LBIMS system is most useful for long-term static storage where traditional containment and surveillance methods are not practical. This combination (long term and static) may be applicable only in limited situations for nuclear material in safeguarded facilities. However, this system may be more useful in non-nuclear arms control applications, such as in monitoring stockpiled weapons and weapon components. 